Urologe 2018·57:1-2

https://doi.org/10.1007/s00120-017-0562-6

Online publiziert: 11. Dezember 2017

(c) Springer Medizin Verlag $\mathrm{GmbH}$, ein Teil von Springer Nature 2017

CrossMark

\author{
V. Stratmann - M. Kretz · P. Herrmann \\ Springer Medizin Verlag GmbH, Heidelberg, Deutschland
}

\title{
Zum Herausgeberwechsel bei Der Urologe
}

können, dass aus der einstigen Vision eine erfolgreiche Realität geworden ist.

Urologe Abschied und Neuanfang. Nach 8 Jahren engagierter und erfolgreicher Amtszeit gibt Herr Prof. Dr. Dr. h.c. Herbert Rübben das Amt des federführenden Schriftleiters von Der Urologe in neue Hände. Gemeinsam mit Herrn Prof. Dr. Gerd Lümmen, wird Herr Prof. Dr. Bernd Wullich zukünftig die Geschicke der Zeitschrift lenken und diese in die Zukunft führen.

Mit dem Abschied aus der Schriftleitung geht für Der Urologe eine Ära zu Ende, die weit über die im Jahre 2010 begonnene Schriftleitung zurückreicht und eng verbunden ist mit der Entwicklungsgeschichte der Zeitschrift aus zwei Schwesternzeitschriften $\mathrm{zu}$ einer großen Zeitschrift für alle Urologen.

Unter der damaligen Schriftleitung bestehend aus den Herren Prof. Knipper, Sökeland und Winz wurde Prof. Rübben schon 1984 als „Fachredakteur", wie es damals hieß, in das Herausgeberboard von Der Urologe B gewählt. In die Schriftleitung trat Prof. Rübben seinerseits 1992 ein. Nachdem Prof. Rübben 1991 auch in das Herausgebergremium von Der Urologe A gewählt wurde, wirkte er bis zum Zusammenschluss der beiden Zeitschriften in beiden Herausgebergremien aktiv mit.

Herrn Prof. Rübben war es ein besonderes Anliegen, eine gemeinsame Fortbildungszeitschrift für alle Urologen herauszubringen. Dieses Anliegen hat er als Mitglied des DGU-Vorstands und besonders in seiner Amtszeit als Generalsekretär ab 1994 und der darauf folgenden Präsidentschaft im Jahr 1999 erfolgreich vertreten, sodass wir heute, 15 Jahre nach der Gründung von Der Urologe, sehen
Nach der Zusammenführung von Der Urologe $A$ und $B$ wurde die federführende Schriftleitung der Zeitschrift paritätisch von der Deutschen Gesellschaft für Urologie und dem Berufsverbands der Deutschen Urologen besetzt. Nachdem Prof. Rübben fast 20 Jahre an der Gestaltung, Weiterentwicklung und damit dem Erfolg von Der Urologe mitgewirkt hatte, übernahm er im Jahr 2010 die Federführung von seinem Vorgänger Prof. Richard Hautmann. Diese übte er zunächst gemeinsam mit Herrn Prof. Mark Goepel und ab 2012 Prof. Gerd Lümmen erfolgreich aus.

Man kann rückblickend konstatieren, dass seine erfolgreiche und stetige publizistische Aktivität für das Fach Urologie einen sehr großen Einfluss hatte. Über 30 Jahre als Herausgeber einer Zeitschrift tätig zu sein, heißt dabei auch Veränderungen in der Wissenschaft, im Publikationswesen und im Informationsbedürfnis der Leser zu begleiten und zu gestalten. Herr Prof. Rübben hat diese Herausforderung mit Bravour gemeistert und die Zeitschrift und das Herausgeberboard dabei immer mit dem richtigen Maß an Selbstbestimmung und Führung geleitet.

Aber nicht nur in Bezug auf die Zeitschrift Der Urologe ist Herr Prof. Rübben ein wichtiger Partner von Springer. Sein Standardwerk Uroonkologie, erscheint seit der Erstauflage 1994 inzwischen in der 6. Auflage. Seit der 5. Auflage bewirbt Springer das Standardwerk mit „Rübben: Uroonkologie - Das Buch, an dem kein onkologisch tätiger Urolo-

\section{Jahre Der Urologe - Eine für Alle}

ge vorbeikommt!" Im Hinblick auf die erfolgreiche Tätigkeit von Herrn Prof. Rübben können wir diesen Leitspruch auf seine Person und alle urologisch tätigen Ärzte ausweiten. Denn wer in den letzten 30 Jahren Der Urologe in der Hand hatte, hat damit auch an seiner wertvollen Arbeit teilhaben dürfen.

Wir bedanken uns mit aller Herzlichkeit bei Herrn Prof. Rübben für die großartige Unterstützung. Wir hoffen, dass Sie uns mit Ihrer Expertise weiterhin treu bleiben werden!

\section{Übergabe des Staffelstabes}

Wir freuen uns, mit Herrn Prof. Bernd Wullich einen würdigen und motivierten Nachfolger gefunden zu haben, der ab sofort das Amt als federführender Herausgeber übernimmt. Im Jahr 2007 wurde er an den Lehrstuhl für Urologie der Friedrich-Alexander-Universität Erlangen-Nürnberg berufen. Der Zeitschrift Der Urologe ist Prof. Wullich mit der Rubrik „Klinische Studien und Forschungsförderung“ schon seit vielen Jahren verbunden. Medizinischer Fortschritt und Innovation sind Herrn Prof. Wullich dabei ein wichtiges Anliegen. In diesem Sinne wurde er schon im Jahr 2007 zum ersten Leiter des neu eingerichteten Ressorts „Forschungsförderung" der DGU gewählt, welches er bis 2015 leitete. In seine Amtszeit fällt auch die Gründung von UroEvidence, einem eigenen Zentrum für Wissenssynthese und Wissenstransfer in der Urologie. Mit systematischen Übersichtsarbeiten soll die gegenwärtig beste wissenschaftliche Evidenz für Entscheidungen in der medizinischen Versorgung von Patienten sowie zur Erstellung von Leitlinien zur Verfügung gestellt werden. Seit 2015 ist 
die Rubrik „Evidenzbasierte Medizin“ erfolgreicher Bestandteil jeder Ausgabe. In Der Urologe hat Prof. Wullich damit bereits seinen Schatten vorausgeworfen. Wir freuen uns auf die Zukunft, auf Innovationen und auf das Weiterführen der Erfolgsgeschichte von Der Urologe.

Für den Springer Medizin Verlag

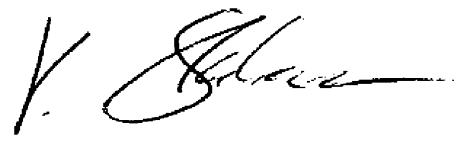

Verena Stratmann

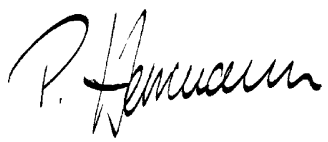

Dr. Paul Herrmann

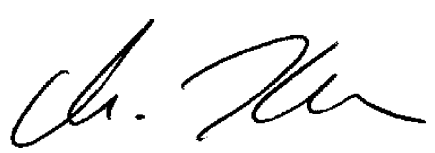

Monika Kretz

\section{Korrespondenzadresse}

\section{Stratmann}

Springer Medizin Verlag GmbH

Tiergartenstr. 17, 69121 Heidelberg,

Deutschland

verena.stratmann@springer.com

Interessenkonflikt. V. Stratmann, M. Kretz und P. Herrmann geben an, dass kein Interessenkonflikt besteht.

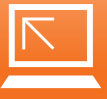

\section{Lesen Sie Der Urologe online auf SpringerMedizin.de}

SpringerMedizin.de bietet Ihnen Zugang zu allen elektronisch verfügbaren Ausgaben und dem CME-Angebot Ihrer Zeitschrift - unabhängig davon, seit wann Sie die Zeitschrift abonniert haben.

So einfach erhalten Sie Zugang zum Online-Archiv:

Registrieren Sie sich einmalig auf www.springermedizin.de/register $\hookleftarrow$ Geben Sie dabei Ihre Einheitliche Fortbildungsnummer (EFN) an.

- Ihr Benutzername entspricht Ihrer E-Mail-Adresse, Ihr Passwort können Sie frei wählen und später jederzeit unter "Mein Profil" ändern.

- Falls Sie bereits ein (Print-) Abonnement bei uns haben, geben Sie bei der Registrierung die Lieferadresse Ihrer Zeitschrift an. Damit wird Ihr Abo-Zugang auf springermedizin.de freigeschaltet.

Sind Sie bereits bei SpringerMedizin.de registriert?

Dann wird Ihr Zeitschriftenabonnement automatisch Ihrem Online-Nutzerkonto hinzugefügt. Sollten die Angaben Ihres Online-Accounts nicht eindeutig mit den Angaben Ihres Zeitschriften-Abonnements übereinstimmen, kann die Zuordnung nicht sicher erfolgen. In diesem Fall und bei allen anderen Fragen zum Online-Zugang kontaktieren Sie bitte unseren Kundenservice unter: Kundenservice@springermedizin.de

Telefonisch erreichen Sie die Hotline montags bis freitags von 9.00 bis $17.00 \mathrm{Uhr}$ kostenfrei unter 0800-77 80777 sowie gebührenpflichtig aus dem Ausland unter +4930884293600.

\section{Der Urologe}

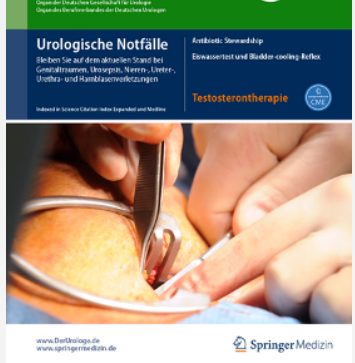

